\title{
In-Motion Ultrasonic Testing of the Tread of High-Speed Railway Wheels using the Inspection System AUROPA III
}

\author{
Hans-Juergen SALZBURGER *; Li WANG, Xiaorong GAO \\ Fraunhofer IzfP*; Photo-electric Engineering Institute of Southwest Jiaotong University \\ Campus E3 1, 66123 Saarbrücken, Germany*; 113\# Box, Southwest Jiaotong \\ University, Chengdu, Sichuan, China 610031 \\ Tel: +49-681-9302 3820*; +86-28-870 9300 \\ E-mail: hans-juergen.salzburger@izfp.fraunhofer.de*; gxrrsclead@126.com
}

\begin{abstract}
The tread of railway wheels underlies high load and wear and its integrity is essential for the safety of the whole train. Visual inspections and optical gauging of the wheel profile are commonly applied for high-speed trains. AUROPA III is a rather new ultrasonic inspection system for the fast and early detection of crack-like defects in the tread of railway wheels. The system enables the inspection of the whole circumference of the tread during passing over stationary ultrasonic probes at speeds up to $15 \mathrm{~km} / \mathrm{h}$. EMAT probes are used which don't require liquid couplings. The ultrasonic wave propagates as a surface wave in the near surface zone of the tread; crack-like defects in this zone are detected by echoes and attenuation of the transmitted wave. This system can inspect all the wheels of a train in a few minutes and allows a fast screening of the condition of the tread. Chengdu Lead Science \& Technology bought this inspection system with the latest state of technology in 2006, evaluated the technique and installed different systems in depots in China. The process of integrating the system into the field application is going on.
\end{abstract}

Keywords: high-speed train, wheel sets, tread inspection, crack-like defects, ultrasonic surface wave, electromagnetic excitation

\section{Introduction}

Non destructive testing (NDT) is an essential issue for all safety relevant components of railway systems. In the case of the rolling stock special attention must be turned to the wheels and axles. Various kinds of NDT-techniques e.g. - ultrasound(UT), eddy current(ET) and magnetic powder (MP) - are used. The full inspection of the wheels can only be applied if the wheel is dismounted from the car or if the wheel set is lifted up and rotated. This means that the inspection can only be done in a wide time raster during the regular inspection intervals after e.g. every $100.000 \mathrm{~km}$ running distance. Early detection is not possible.

The tread of the wheels of High-Speed Trains underlies high load and wear; its integrity is essential for the safety of the whole train. Crack-like defects in the surface cannot be excluded; due to their safety criticality they must be detected at an early stage. The way to achieve this is to perform the inspection as often as possible. This can only be done by techniques which allow the measurements 'in-motion'.

Such 'In-Motion' techniques are available for the gauging of the wheel profile by optical means and applied for High-Speed Trains. AUROPA III is a rather new ultrasonic inspection system for the fast and early detection of crack-like defects in the tread of railway wheels. It enables the inspection of the whole circumference of the tread during passing over stationary 
ultrasonic probes at speeds up to $15 \mathrm{~km} / \mathrm{h}$. EMAT probes are used which don't require liquid couplings.

The system was developed by Fraunhofer-Institute for NDT in Saarbruecken, Germany ${ }^{[1]}$ and introduced in the field together with Deutsche Bundesbahn ${ }^{[2]}$. Chengdu Lead Science \& Technology, Chengdu, China bought this inspection system with the latest state of technology in 2006, evaluated the technique and installed different systems in depots in China.

This paper describes the principle of the inspection technique and presents the system. The system capabilities are presented including examples of the field installations in the PR China.

\section{Inspection-Principle}

The inspection technique is the ultrasonic pulse-echo and pulse transmission technique using Rayleigh waves propagating in circumferential direction along the tread of a railway wheel. These waves are excited and detected by four EMAT probes, mounted at the head of the rails of a special inspection track for the in-motion inspection ( 2 on each rail).

When a train passes the inspection track special sensors detect the arrival of a wheel and recognize when the wheel is in the most close contact with the probe. At this time the ultrasonic surface wave pulse (Rayleigh wave) is transmitted (Figure 1).

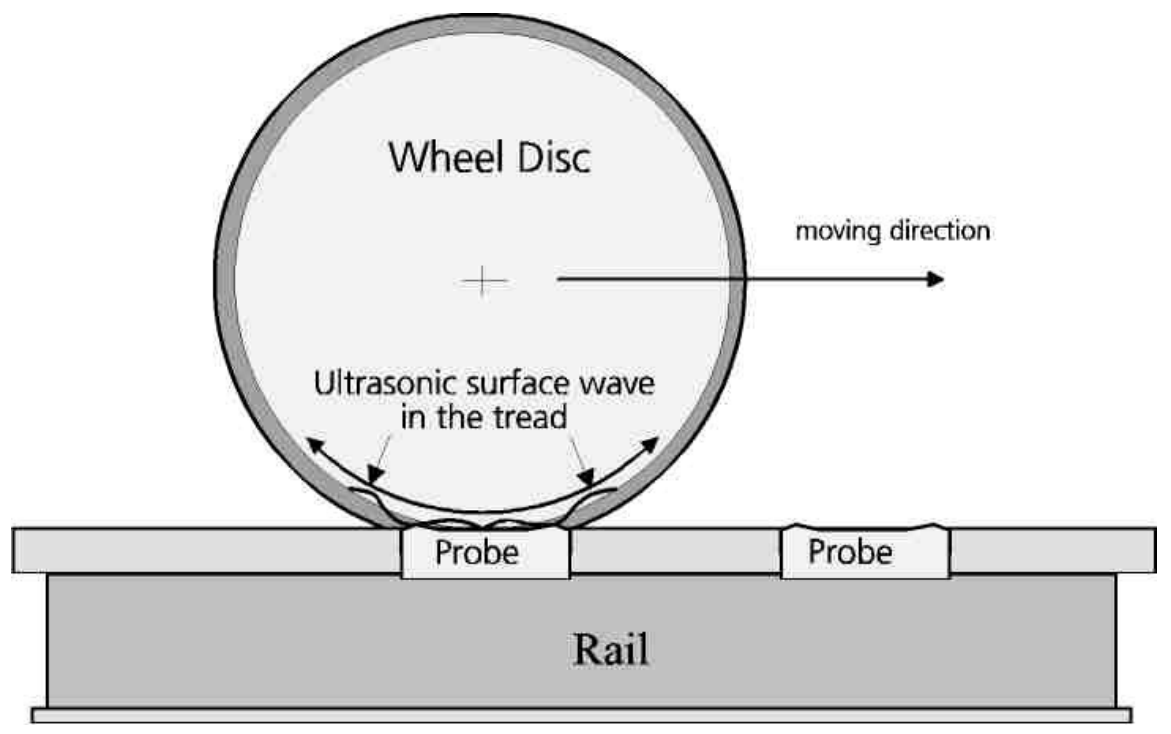

Figure 1: In-motion wheel inspection principle - wheel in contact with the probe

This ultrasonic wave pulse travels within a couple of ms with several round trips (RT) along the tread of the wheel. The probe detects either echoes (E) from discontinuities in the tread (crack-like surface breaking or subsurface defects) in the pulse echo mode or the through-transmission signals after several round trips (RT) (Figure 2). As well the normalized amplitudes of the echoes (E) as the attenuation of the sequence of the RT-signals (RT) are evaluated. 

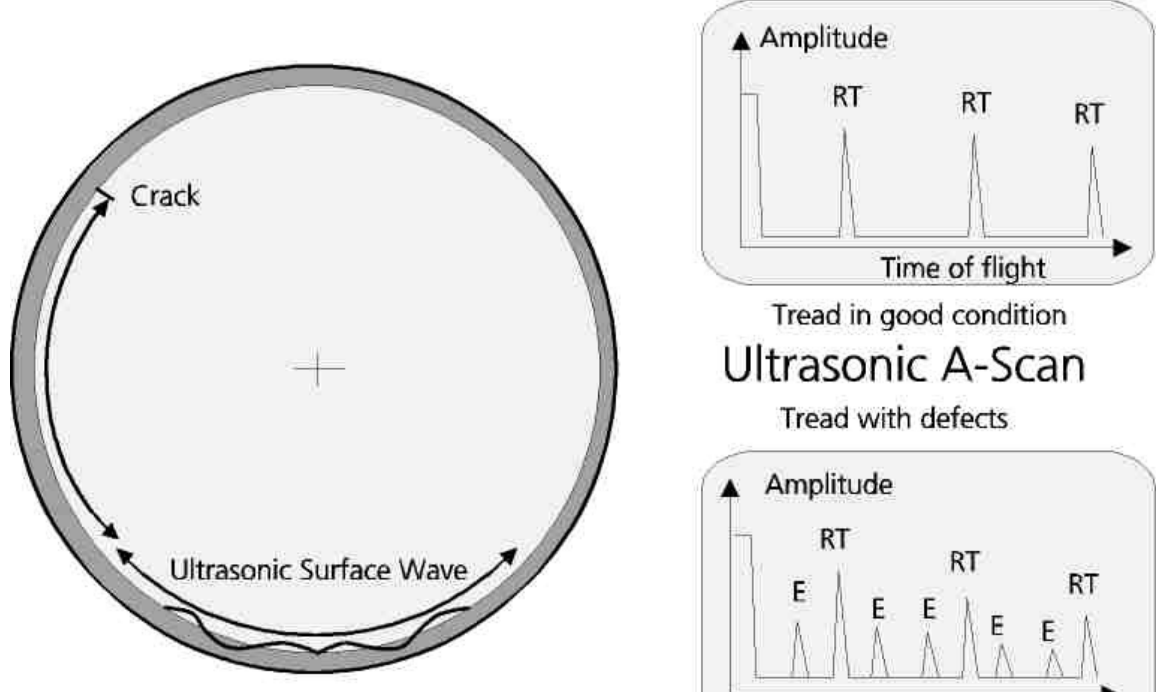

Tread with defects

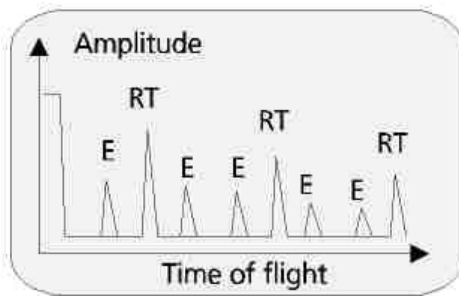

Figure 2: Inspection result (schematic presentation of typical Ultrasonic A-Scan)

\section{Inspection system}

Figure 3 shows a scheme of the complete inspection system. It consists of three components

- The inspection track (to be provided/prepared by the customer)

- The probes

- The Ultrasonic Electronics-/Control-Unit

- Sensors for the control of the operational sequence of the four probes

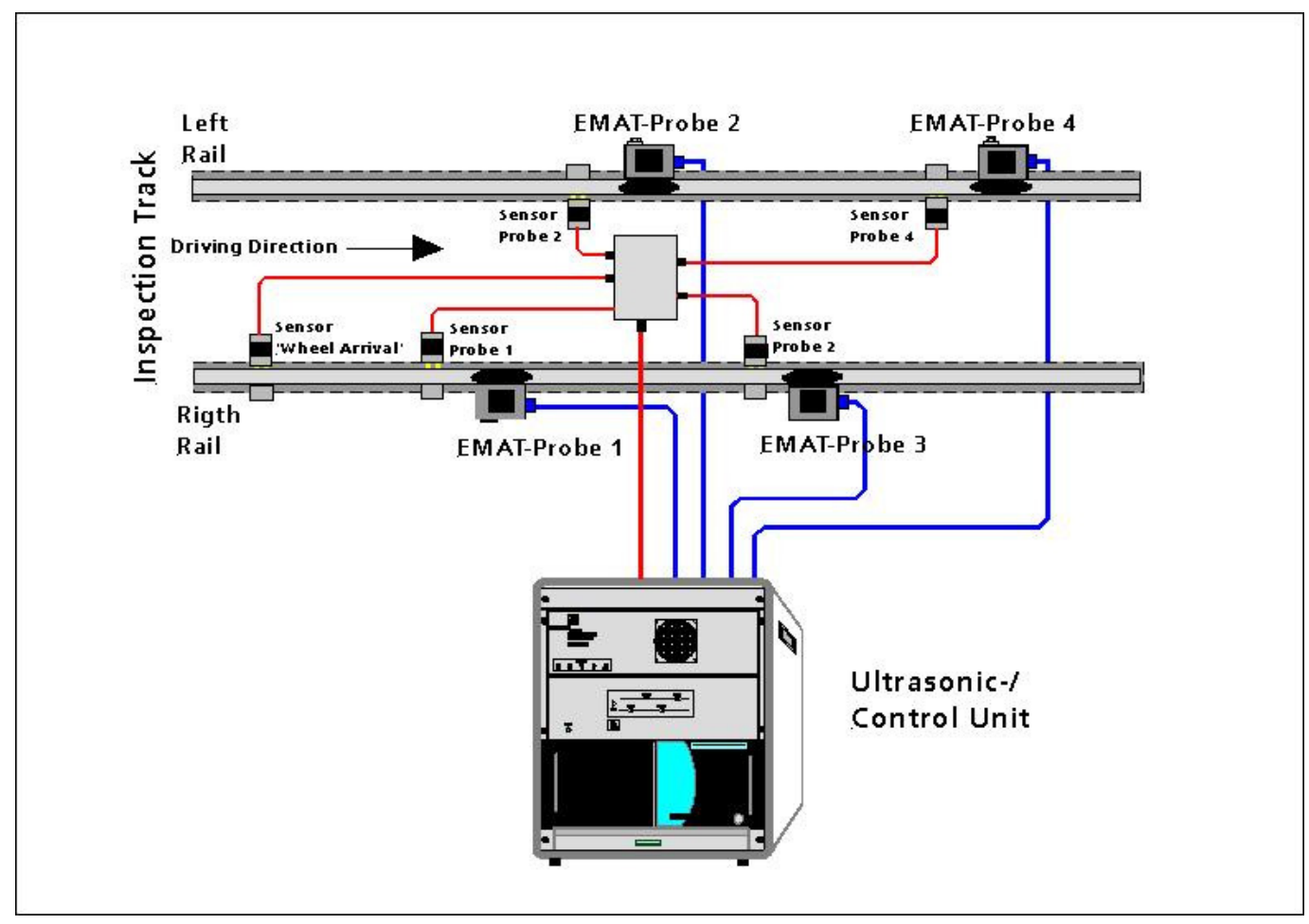

Figure 3: Scheme of the inspection system AUROPA III 


\subsection{Inspection track}

All measurements are performed using a special inspection track. This track consists of two machined rails for the attachment of the probes and of the sensors. The four probes are arranged in such a way that each wheel is inspected two times - each time with a different point of insonification (along the circumference of the wheel) of the ultrasonic wave. Additionally the Inspection Track is equipped with sensors, which are responsible for generating an 'Inspection Start' signal and for counting of the axles and for the triggering of the transmitter-electronics.

Manufacturing and installation of the track are specific for each customer and their infrastructure conditions (necessary civil work etc.). Details and prices of the track can only be given if the necessary information is available.

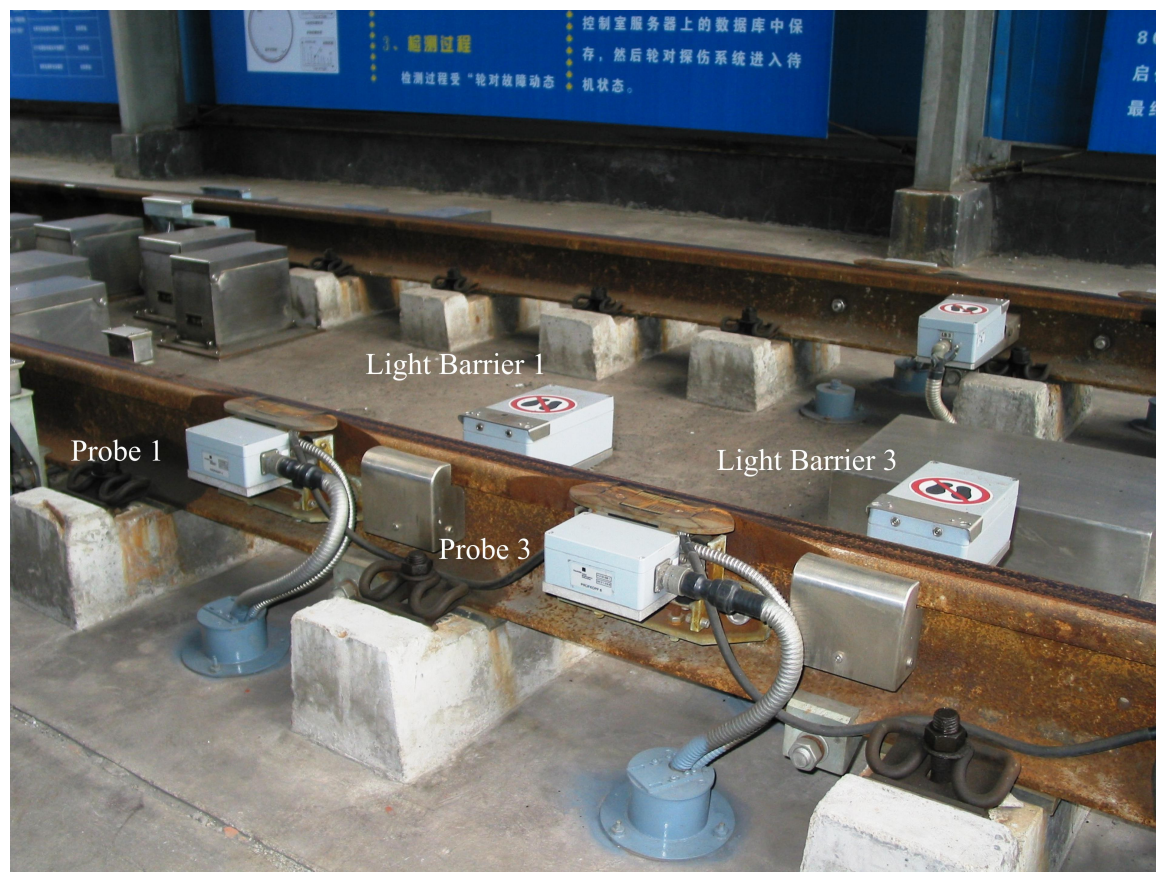

Figure 4: Inspection Track with two probes and the corresponding light barrier sensors

(Depot Quingdao)

\subsection{Probes}

The probes are working free of couplant according to the EMAT-principle. They consist of an DC-electromagnet generating a magnetic field normal to the wheel surface and a RFcoil for generating and receiving the ultrasonic wave.

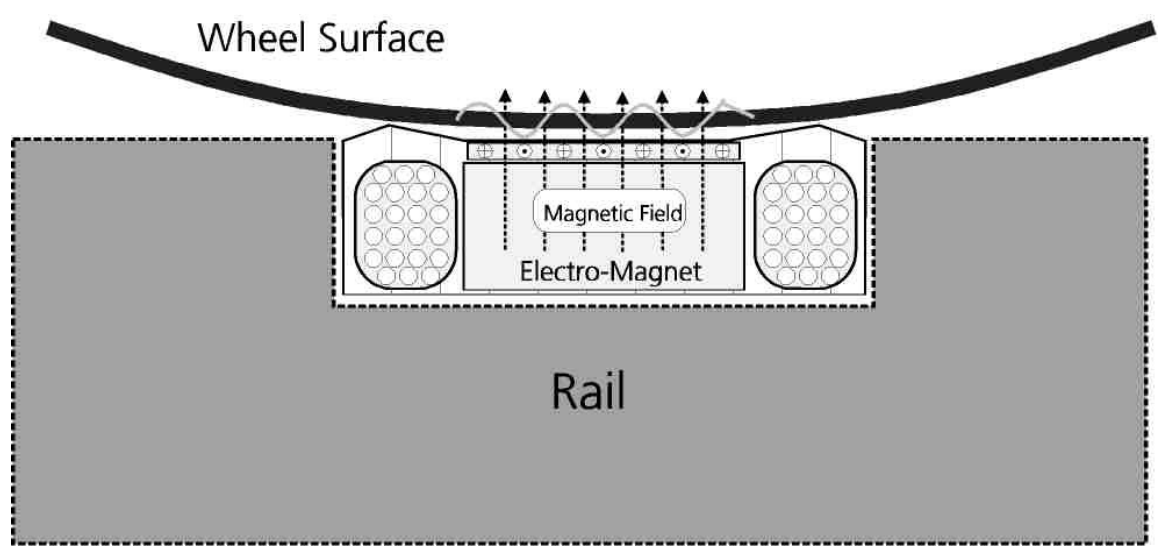

Figure 5: Probe design 
The probes are shown in more detail in Figure 6, which is a top view onto the probe; the grey box contains the preamplifier and the connector to the Ultrasonic-/Control Unit. The probe is fixed by a holder which can be easily attached to the rail web by a slightly movable suspension.

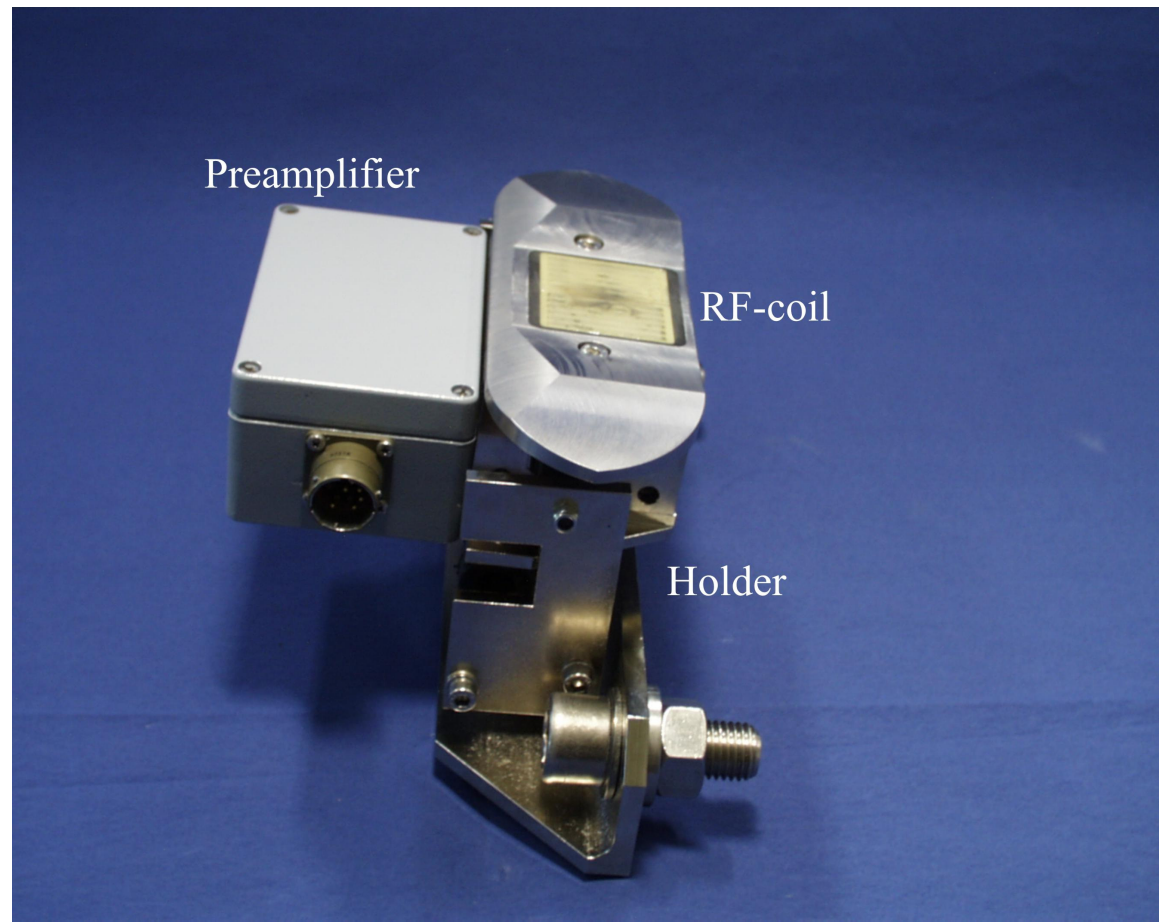

Figure 6: Probe with housing, preamplifier and holder

\subsection{Ultrasonic Electronics Control Unit}

The two hardware-components - Ultrasonic Electronics-/Control Unit and PC RI 25 Simatic are assembled together with other hardware components in a $19^{\prime \prime}$ rack.

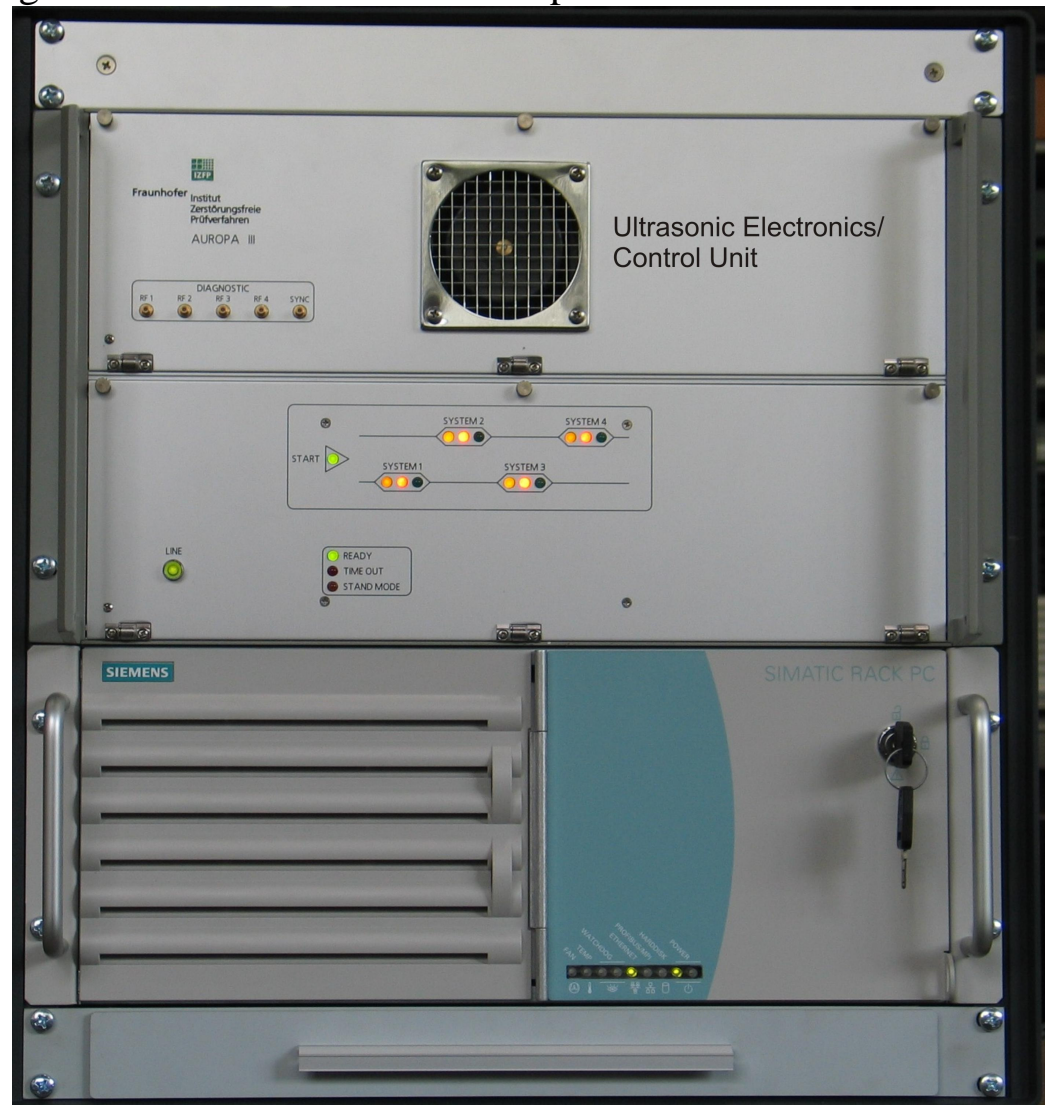


Figure 7: Ultrasonic Control Unit

The operation of the ultrasonic electronics-/control unit is performed by the PC via a TCP/IP interface.

A Digital I/O Board acquires the signals of the light barrier sensors of the inspection track and displays the status of the system on the front panel of the ultrasonic electronics by a couple of LED's. The CPU Board generates the system trigger by the , synchronizes the ADC and delivers a square wave burst signal to the transmitter amplifier. The received signal is filtered and amplified by the built in preamplifier and transferred to the ADC.

\subsection{System capabilities}

AUROPA III is designed to fulfill the following technical and inspection requirements:

Ultrasonic pulse echo and pulse-through transmission Inspection of the tread of railway wheels using RAYLEIGH-surface waves

Inspection range: Double circumference of the wheel (up to 7 meters)

Patented Electromagnetic Probes (EMAT) for bidirectional inspection

In-Motion - Inspection of all wheels of a train and of single axes using one A-Scan per probe

Stationary Inspection of a single wheel (axis) with a pulse repetition frequency of $10 \mathrm{~Hz}$

Twofold in-motion Inspection of each wheel with two separate probes from different points of insonification along the circumference of the wheel

Software control of the ultrasonic parameters, the trigger parameters and the light barriers

On-line record and storage of the A-Scan's

Off-line Display of the recorded A-Scan's

Inspection only possible in one direction of the (driving direction )

Material of the wheels to be inspected: Ferromagnetic carbon steel grades (rolled or forged)

Speed of the train or wheel during inspection:

Maximal load per wheel set:

$5-15 \mathrm{~km} / \mathrm{h}$

Range of the width of the running surface: $\quad 80-140 \mathrm{~mm}$

Range of the diameter of the wheel: $\quad 600-1300 \mathrm{~mm}$

Minimal Spacing between two wheel-sets: $\quad 1.5 \mathrm{~m}$

AUROPA III achieves the following inspection results:

- Artificial Defect 1: side drilled hole with a diameter of $2 \mathrm{~mm}$, a length of $40 \mathrm{~mm}$ in parallel to the running surface in a depth of $3 \mathrm{~mm}$ below the running surface.

- Artificial Defect 2: Saw cut with a length of $45 \mathrm{~mm}$ in transversal direction to the circumferential direction (maximum depth: $\cong 3 \mathrm{~mm}$ ).

\section{Application Example}

From the end of the year 2005, AUROPA III was installed and put into use at Zhengzhou Depot. In the following year, more than 10 defects in the tread of the wheel sets have been detected by the inspection system.

Figure 8 shows one of the defects on the tread of a wheel set (loss of material) which was detected by AUROPA III. The locomotive is $\mathrm{DF}_{11 \mathrm{G}} 0144$ which is belonging to the Xuzhou depot of Jinan Railway administration. The corresponding ultrasonic A-Scan of this wheel set (axis 1) recorded by probe 4 is displayed in Figure 9. This dialogue window contains besides the A-Scan information about the number of the inspected axis, the probe generating the corresponding A-scan and date and time of the measurement. 


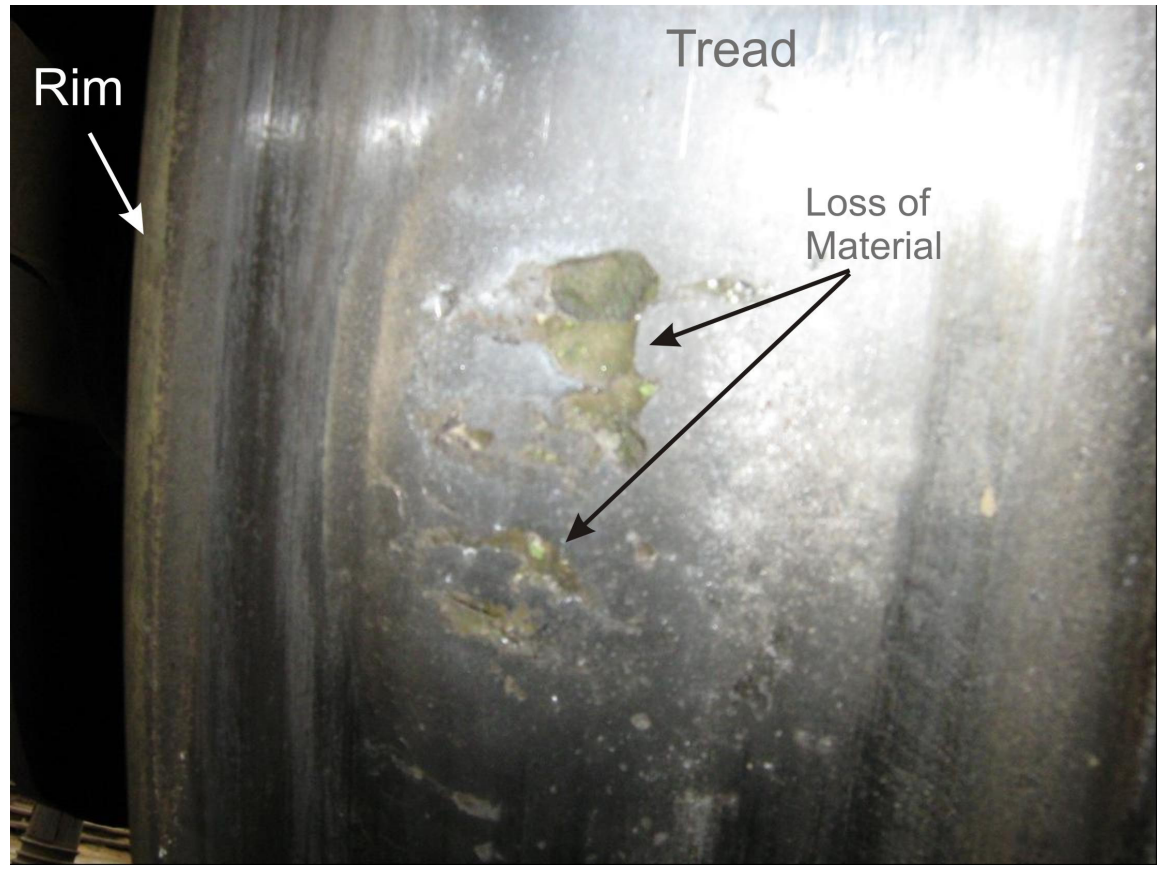

Figure 8: Defect (loss of material) detected by AUROPA III

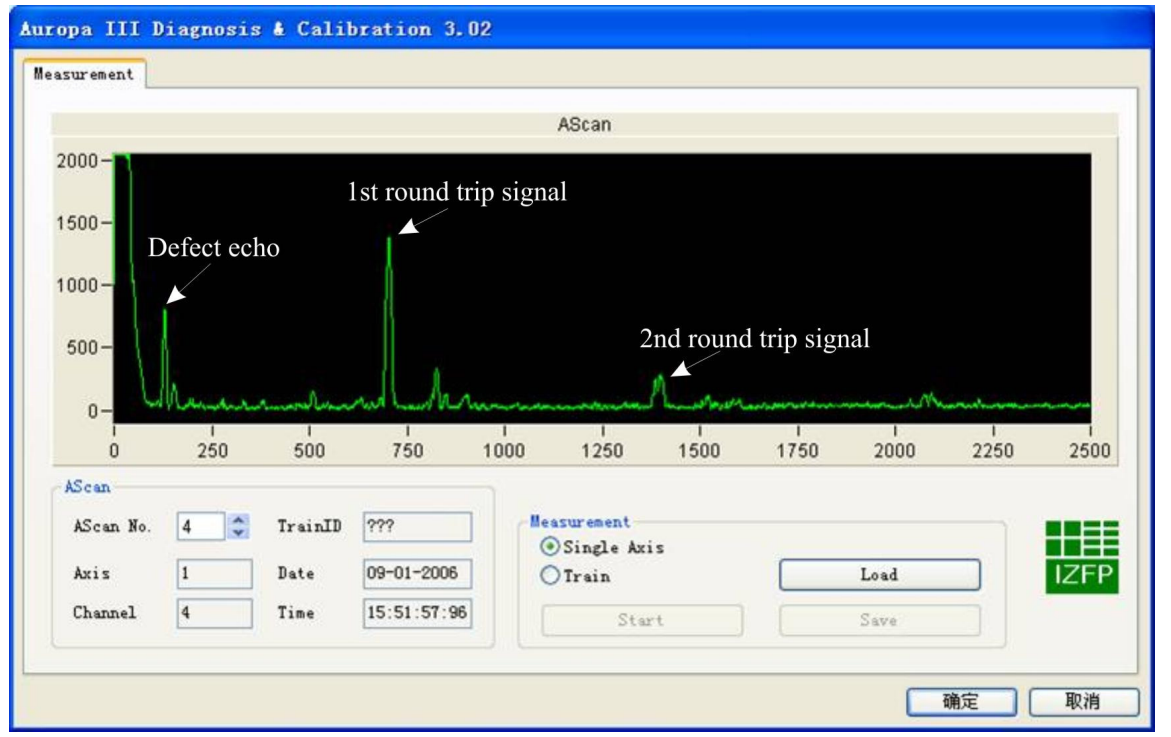

Figure 9: Ultrasonic A-Scan of the wheel with defect shown in Figure 8

One example of the instalment of Auropa III for the inspection of High-Speed trains operated by Chinese railway administration is given in the picture of Figure 10. It shows the train $\mathrm{CRH} 2$ approaching the inspection unit in Beijing EMU depot. 


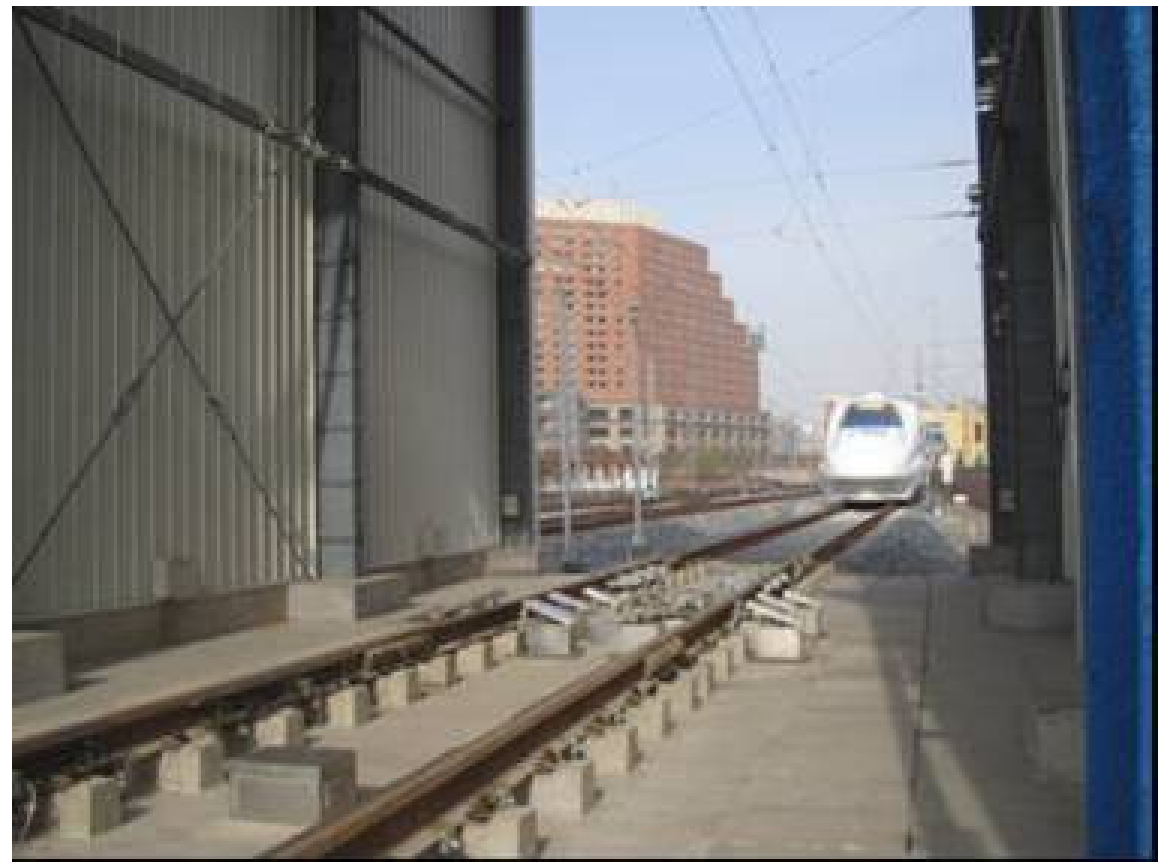

Figure 10: Inspection track at Beijing EMU depot

\section{References}

[1] Salzburger, H.J., Repplinger, W.: Automatic in-motion inspection of the tread of railway wheels by e.m.a. excited Rayleigh waves

Ultrasonics International 1983 : Conference Proceedings. Guildford : IPC Science and Technology Press, 1983.

[2] Salzburger, H.J., Repplinger, W., Schmidt, W.: Entwicklung, Betriebstest und Einsatz eines automatischen Systems zur wiederkehrenden Prüfung der Laufflächen von EisenbahnRädern

Deutsche Gesellschaft für Zerstörungsfreie Prüfung e.V. (DGZfP): Aktuelle Aufgaben der zfP: Bewährte Aufgaben - neue Wege. Teil 1. Berlin : Deutsche Gesellschaft für zerstörungsfreie Prüfung (DGZfP), 1987. 